\title{
Diffusion Tensor Mapping of the Human Brain Using Single-Shot Line Scan Imaging
}

\author{
Jürgen Finsterbusch, $\mathrm{PhD}$, and Jens Frahm, $\mathrm{PhD}^{*}$
}

\begin{abstract}
A recently developed single-shot line scan imaging technique for diffusion measurements (Finsterbusch and Frahm, Magn Reson Med 1999;42:772-778) was extended to full diffusion tensor mapping of the human brain. Because the sequence acquires stimulated echoes from individual columns of magnetization ("lines"), the approach is affected neither by spatial aliasing when studying inner volumes nor by resonance offset effects or $\mathrm{T2}^{*}$ dephasing as in diffusion-weighted echoplanar imaging. Experiments on healthy subjects were performed at $2.0 \mathrm{~T}$ using 31 single-shot images ( $5 b$ values, 6 orientations, $520 \mathrm{msec}$ each) at $1.5 \times 1.5 \mathrm{~mm}^{2}$ resolution (interpolated) and $6.0 \mathrm{~mm}$ section thickness. Apart from calculated images with isotropic diffusion weighting, the results include maps of the six independent diffusion tensor components, the apparent diffusion coefficient, the relative anisotropy, and the main diffusion direction. The achievable signal-to-noise ratio and resolution allow the identification of differently oriented nerve fibers in the brain stem. J. Magn. Reson. Imaging 2000;12:388-394. () 2000 Wiley-Liss, Inc.
\end{abstract}

Index terms:magnetic resonance imaging; diffusion tensor; diffusion anisotropy; human brain; human brain stem; line scan imaging

BECAUSE OF THE MICROSCOPIC structural heterogeneity of living tissue, water diffusivity is a directiondependent quantity that must be described by a symmetric tensor D containing six independent elements:

$$
\mathbf{D}=\left(\begin{array}{lll}
D_{x x} & D_{x y} & D_{x z} \\
D_{x y} & D_{y y} & D_{y z} \\
D_{x z} & D_{y z} & D_{z z}
\end{array}\right)
$$

Access to the diffusion tensor by suitably encoded diffusion-weighted (DW) MRI may be exploited in several ways. For example, because the predominant diffusion direction within a white matter voxel mainly characterizes the orientational coherence of nerve fibers $(1,2)$, such information may be used for fiber tracking in the living human brain (3). Moreover, the relative anisot-

Biomedizinische NMR Forschungs GmbH, 37070 Göttingen, Germany. Presented in part at the 7th and 8th Scientific Meetings of the International Society for Magnetic Resonance in Medicine, Philadelphia, 1999, and Denver, 2000.

*Address reprint requests to: J.F., Biomedizinische NMR Forschungs GmbH, 37070 Göttingen, Germany.

E-mail: jfrahm@gwdg.de

Received December 27, 1999; Accepted May 2, 2000. ropy of water diffusion has been suggested to reflect the degree of myelination and therefore may help to study pertinent disorders (4). On the other hand, clinical applications to cerebral ischemia mostly aim at diffusion parameters that do not rely on the relative orientation of the tissue and the diffusion gradients. Thus, a popular invariant of the diffusion tensor is its trace or apparent diffusion coefficient (ADC), here defined as

$$
\operatorname{ADC}=\frac{1}{3} \operatorname{Trace}(\mathbf{D})=\frac{1}{3}\left(D_{x x}+D_{y y}+D_{z z}\right)
$$

In this form the ADC may be used to obtain an isotropic DW image for a rapid and reliable assessment of patients with acute stroke (5).

So far, most measurements of the diffusion tensor have been performed with echoplanar imaging (EPI). However, EPI artifacts caused by chemical shift effects, $T 2 *$ signal dephasing, and distorted phase encoding in the presence of susceptibility differences severely hamper its application to a variety of brain regions. Complementing DW sequences based on spin echoes $(6,7)$, the present study extends a recently developed technique (8) for DW single-shot line scan imaging (LSI) to full diffusion tensor mapping of the human brain. Because the approach is based on a high-speed acquisition of stimulated echoes, the resulting diffusion maps are insensitive to the aforementioned gradient-echo drawbacks. The potential of diffusion tensor mapping using single-shot LSI is demonstrated by its capability to identify nerve fibers in the brain stem.

\section{MATERIALS AND METHODS \\ DW Single-Shot LSI}

The basic pulse sequence for DW single-shot LSI of a single slice is shown in Fig. 1. The first two radiofrequency (RF) pulses generate a spin echo (SE) encompassing the DW gradients. It replaces the free induction decay following the first $\mathrm{RF}$ pulse of a conventional single-shot STEAM sequence (9). The subsequent sliceselective RF pulse flips the DW magnetization back into the longitudinal direction. Finally, line-selective stimulated echoes (STEs) are generated by a series of spatially shifted RF excitations. To achieve a columnar cross section, the orientation of the line selection gradient is chosen perpendicular to the previously defined 


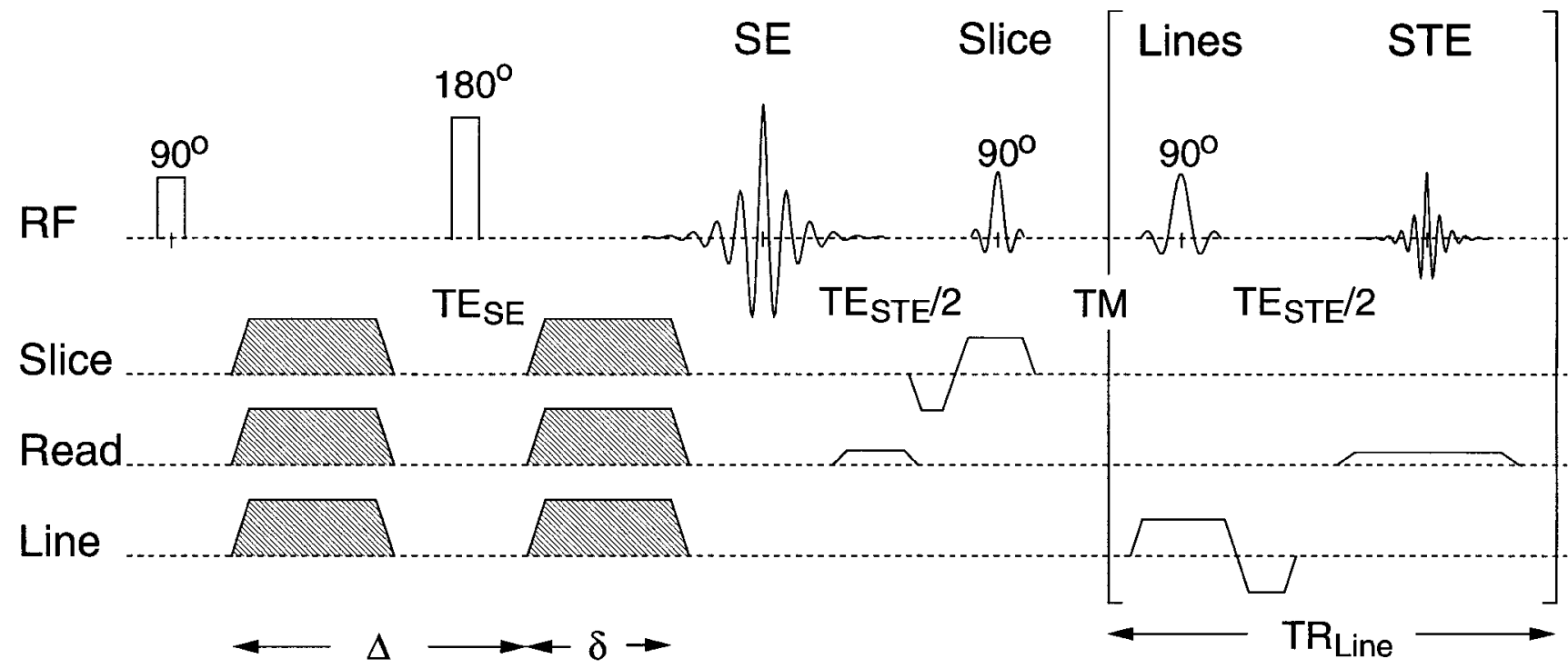

Figure 1. Basic MRI sequence for diffusion-weighted single-shot line scan imaging (DW LSI) using stimulated echoes (STE). The bracket must be repeated with spatially shifted excitations to obtain a full image. The field of view is given by the number of lines acquired.

Figure 2. Diffusion tensor mapping of the human brain. Top left: Calculated image without diffusion-weighting. Top right: Isotropic DW image for $b=1000 \mathrm{sec} \mathrm{mm}^{-2}$. Bottom left: apparent diffusion coefficient (ADC) map. Bottom right: Relative anisotropy (RA) map. DW single-shot line scan images were acquired within $520 \mathrm{msec}$ (40 lines) at $3.0 \times 3.0 \mathrm{~mm}^{2}$ resolution (interpolated to $1.5 \times 1.5 \mathrm{~mm}^{2}$ ) and $6 \mathrm{~mm}$ section thickness. The total measurement time was 3 minutes 16 seconds using $5 b$ factors, 6 orientations, and a repetition time of 6.5 seconds.

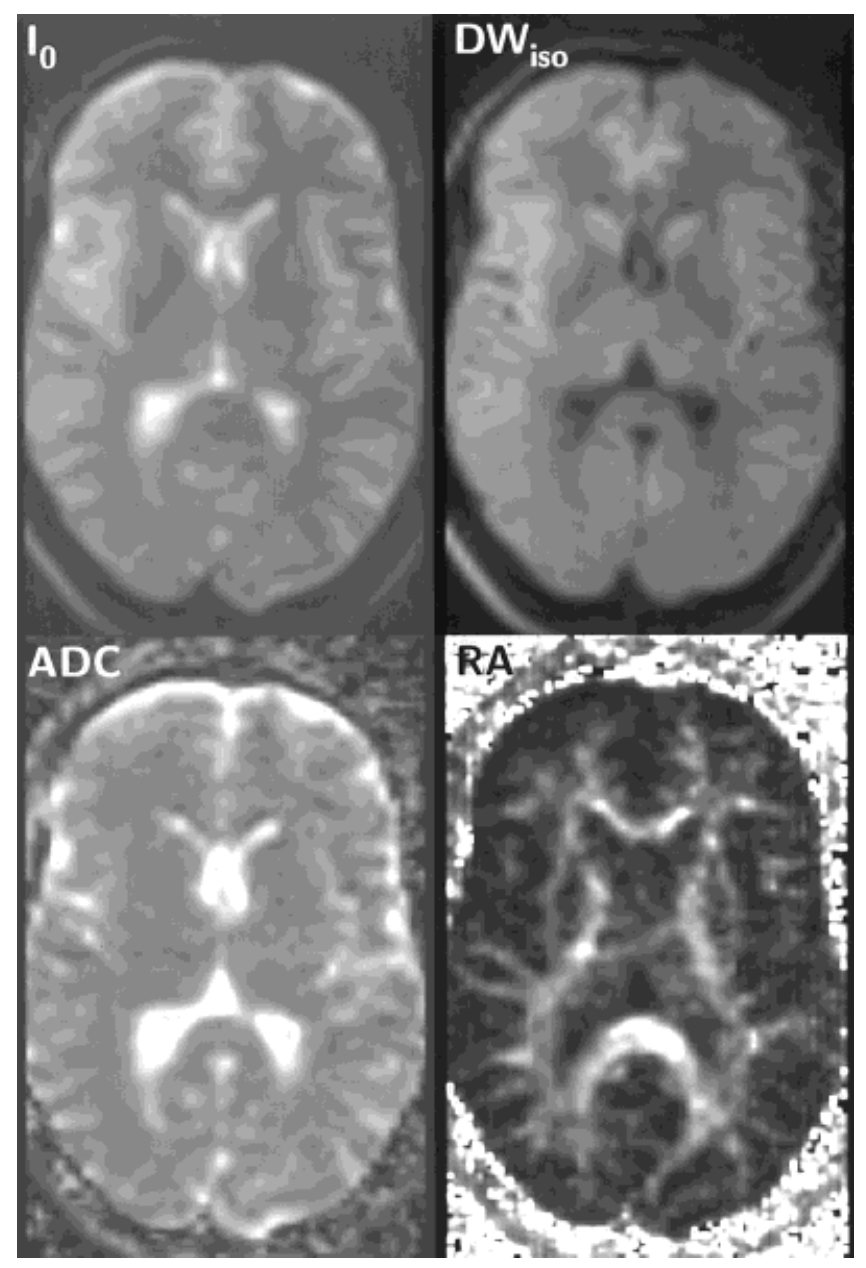




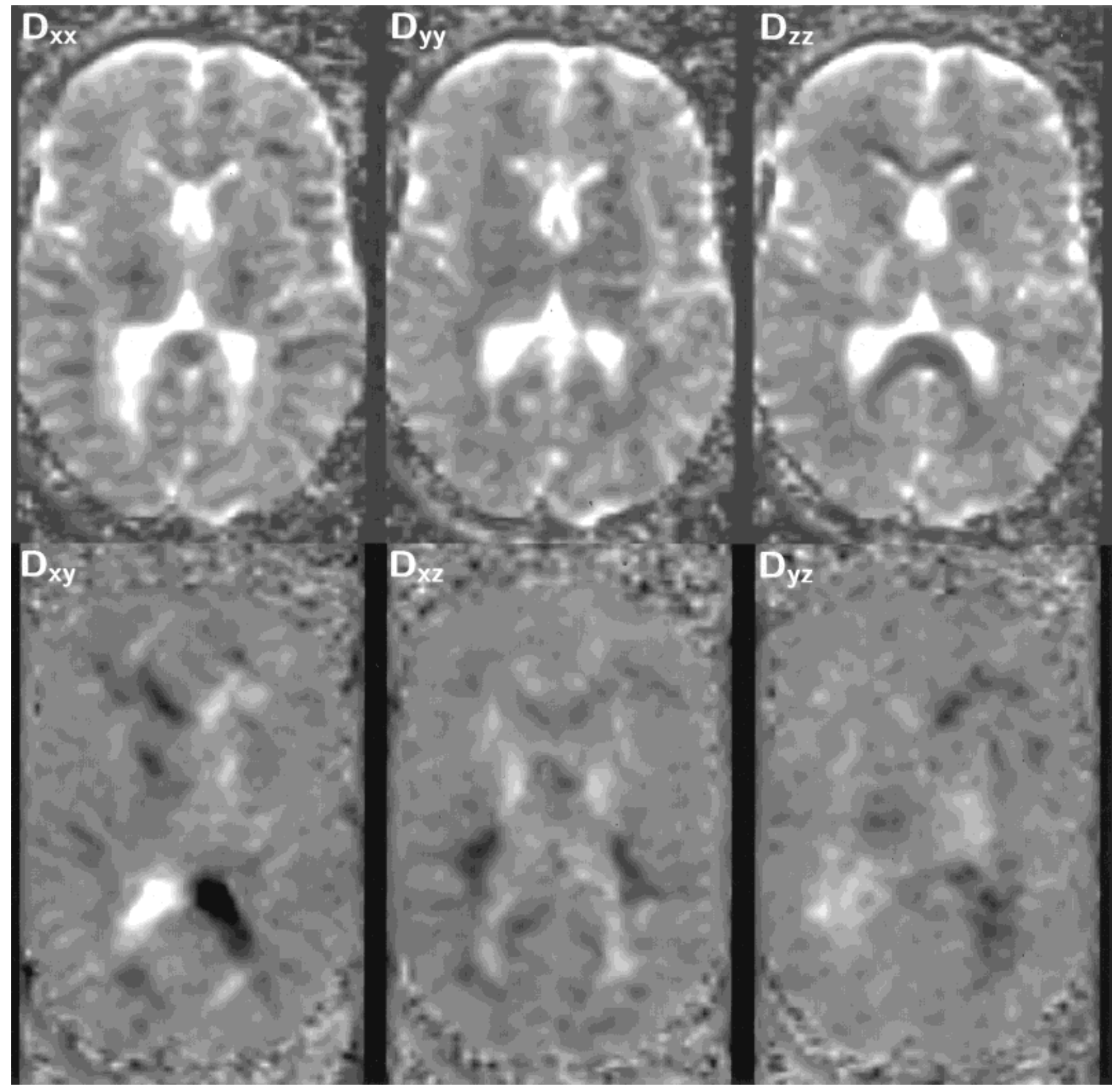

Figure 3. Diffusion tensor mapping of the human brain (same data and parameters as in Fig. 2). The maps represent the six elements of the diffusion tensor.

plane. For image reconstruction the STE signals are Fourier transformed and stored as consecutive intensity profiles in an image matrix. Because the magnetization of each line is excited only once, the "read" RF pulses can have flip angles of $90^{\circ}$.

To enhance the diffusion-encoding efficiency, DW gradients were based on a combination of two physical gradients. In accordance with to ref. 2, we employed $\mathrm{read} /$ line/slice gradient ratios of 1:1:0, 1:1:0, 1:0:1, $1: 0: \overline{1}, 0: 1: 1$, and $0: 1: \overline{1}$. All six orientations were measured for five $b$ factors, ie, 510, 617, 735, 862, and 1000 sec $\mathrm{mm}^{-2}$, covering a wide range of signal intensities and diffusivities. Thus, including one image without DW, each data set, ie, each section, required the acquisition of 31 single-shot images.

\section{Analysis of Diffusion Parameters}

The calculation of diffusion tensor maps was based on a pixel-by-pixel multivariable regression analysis minimizing the sum of the squared deviations of the measured pixel intensities from the model parameters $I_{0}$ (ie, the image intensity without DW) and $D_{i j}(i, j=x, y, z)$. Each of the terms was weighted with its corresponding pixel intensity to correct for the nonlinear behavior of the transformation used $l n$, eg, compare ref. 2.

An appropriate algorithm was added to the standard image processing software of the scanner for automated online computations. In addition to maps representing $I_{0}$ and the six tensor elements $D_{i j}$, the analysis included the calculation of an ADC map, an isotropic DW image 


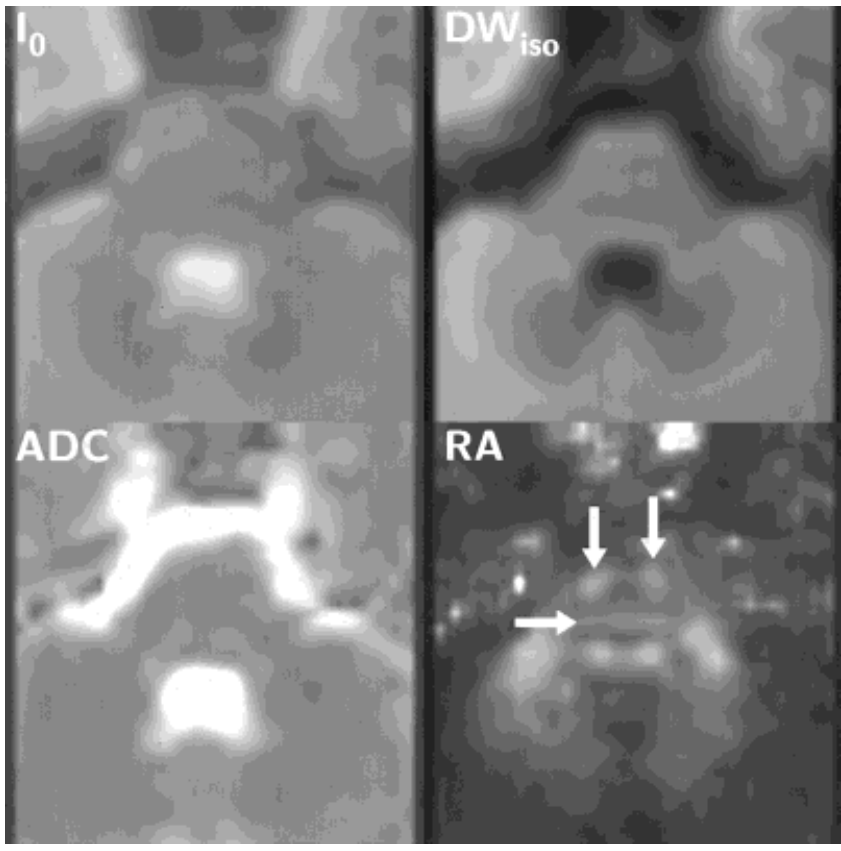

Figure 4. Diffusion tensor mapping of the human brain stem. Top left: Calculated image without diffusion-weighting. Top right: Isotropic DW image for $b=1000 \mathrm{sec} \mathrm{mm}^{-2}$. Bottom left: Apparent diffusion coefficient $(\mathrm{ADC})$ map. Bottom right: relative anisotropy (RA) map. The total measurement time was 1 minutes 46 seconds using a repetition time of 3.5 seconds. The field of view required only 25 lines, which allowed us to increase the receiver bandwidth while keeping the imaging time constant. The arrows in the RA map indicate nerve fiber tracts with different orientations (see text).

at $b=1000 \mathrm{sec} \mathrm{mm}^{-2}$, and a normalized relative anisotropy (RA) map according to ref. 1:

$$
\mathrm{RA}=\frac{1}{\sqrt{6}} \frac{\sqrt{\sum_{i}\left(\mathrm{ADC}-D_{i i}\right)^{2}+2\left(D_{x y}^{2}+D_{x z}^{2}+D_{y z}^{2}\right)}}{\mathrm{ADC}} .
$$

To enhance the readibility of the tensor maps, further postprocessing involved calculation of the main diffusion direction (MDD), ie, the direction of the largest eigenvalue of the diffusion tensor. Pertinent information was color-coded and superimposed onto the original RA maps using an empirical threshold of RA $>0.22$.

\section{Experimental Studies}

Studies of young healthy volunteers were performed at $2.0 \mathrm{~T}$ (Magnetom Vision, Siemens, Germany) equipped with a conventional $25 \mathrm{mT} \mathrm{m}^{-1}$ gradient system (maximum slew rate $40 \mathrm{~T} \mathrm{~m}^{-1} \mathrm{~s}^{-1}$ ) and the standard head coil. Written informed consent was obtained in all cases before the examination.

Transverse brain sections of $6 \mathrm{~mm}$ thickness were covered with line excitations proceeding from left to right. The in-plane resolution of $3.0 \times 3.0 \mathrm{~mm}^{2}$ was interpolated to $1.5 \times 1.5 \mathrm{~mm}^{2}$ during image reconstruction. Taking into account limitations of the duty cycle, the maximum amplitude of the DW gradients was set to $17.9 \mathrm{mT} \mathrm{m}^{-1}$. Thus, for a maximum $b$ value of $1000 \mathrm{sec}$ $\mathrm{mm}^{-2}$, a gradient duration of $\delta=29.7 \mathrm{msec}$ and a diffusion time of $\Delta=34.6 \mathrm{msec}$ resulted in a spin-echo time of $\mathrm{TE}_{\mathrm{SE}}=66 \mathrm{msec}$.

The field-of-view (FOV) in-line direction was adapted to the region of interest. This increases the signal-tonoise ratio (SNR) because a lower number of lines allows for a smaller receiver bandwidth, ie, a longer acquisition period per echo, for a given total imaging time and respective $\mathrm{T} 1$ attenuation. Accordingly, measurements of the cerebrum involved a $120 \mathrm{~mm}$ FOV (40 lines), an echo acquisition time of $T_{\text {acq }}=7.3 \mathrm{msec}$, an STE time of $\mathrm{TE}_{\mathrm{STE}}=10.5 \mathrm{msec}$, and a line repetition time of $\mathrm{TR}_{\text {Line }}=10.5 \mathrm{msec}$, which translates into a measuring time of $520 \mathrm{msec}$ per single-shot image. The measurement time for full diffusion tensor mapping was 3 minutes 16 seconds for a TR of $6500 \mathrm{msec}$.

The brain stem was covered by a much smaller FOV of $75 \mathrm{~mm}$ (25 lines). Using $T_{\mathrm{acq}}=12.5 \mathrm{msec}, \mathrm{TE}_{\mathrm{STE}}=$ $15.8 \mathrm{msec}$, and $\mathrm{TR}_{\text {Line }}=15.8 \mathrm{msec}$ resulted in a measuring time of $476 \mathrm{msec}$ per image. The total measurement time for diffusion tensor mapping was 1 minute 44 seconds for a TR of 3500 msec. For both large and small FOVs, the echo acquisition period $T_{\text {acq }}$ was optimized as described in ref. 8 to maximize the available SNR for white matter and the last line of the image, which suffers the strongest $\mathrm{T} 1$ attenuation.

\section{RESULTS}

Figures 2 and 3 summarize the results of a full diffusion tensor measurement for a transverse brain section at the level of the ventricles. Whereas Fig. 2 presents the calculated $I_{0}$ image, an isotropic DW image, and maps of the ADC and RA, Fig. 3 depicts maps of the individual tensor elements. Together with the orientation-invariant RA map, which identifies areas of marked anisotropy, the tensor elements allow a detailed assessment of the direction dependency of tissue water diffusion. For instance, in the medial part of the corpus callosum, diffusion occurs mainly in the left-right direction, which is best appreciated in the $D_{y y}$ map. However, the lateral ends of the corpus callosum are highlighted in the $D_{x y}$ map, yielding strong positive and negative amplitudes, as expected from the changed fiber orientations. In general, the results confirm that the diffusion anisotropy is most pronounced in white matter, especially in regions with a preferred fiber orientation such as the corpus callosum or the internal capsule. Pertinent areas correspond to high intensities in the RA map despite a rather uniform distribution of diffusion coefficients, as seen in the corresponding ADC map (Fig. 2).

Similarly, Figs. 4 and 5 present the results of a diffusion tensor study of the human brain stem for a section covering the pons and part of the cerebellum. Again, in striking difference to the absence of any signal heterogeneity ("contrast") in the ADC map, the RA map of the pons identifies distinct regions with high diffusion anisotropy (arrows in Fig. 4). An interesting feature is their different directional behavior, as revealed by the individual tensor element maps and the color-coded MDD map indicating the main diffusion direction (Fig. 5). In regions marked with vertical arrows, diffusion is predominantly perpendicular to the section (blue to purple 

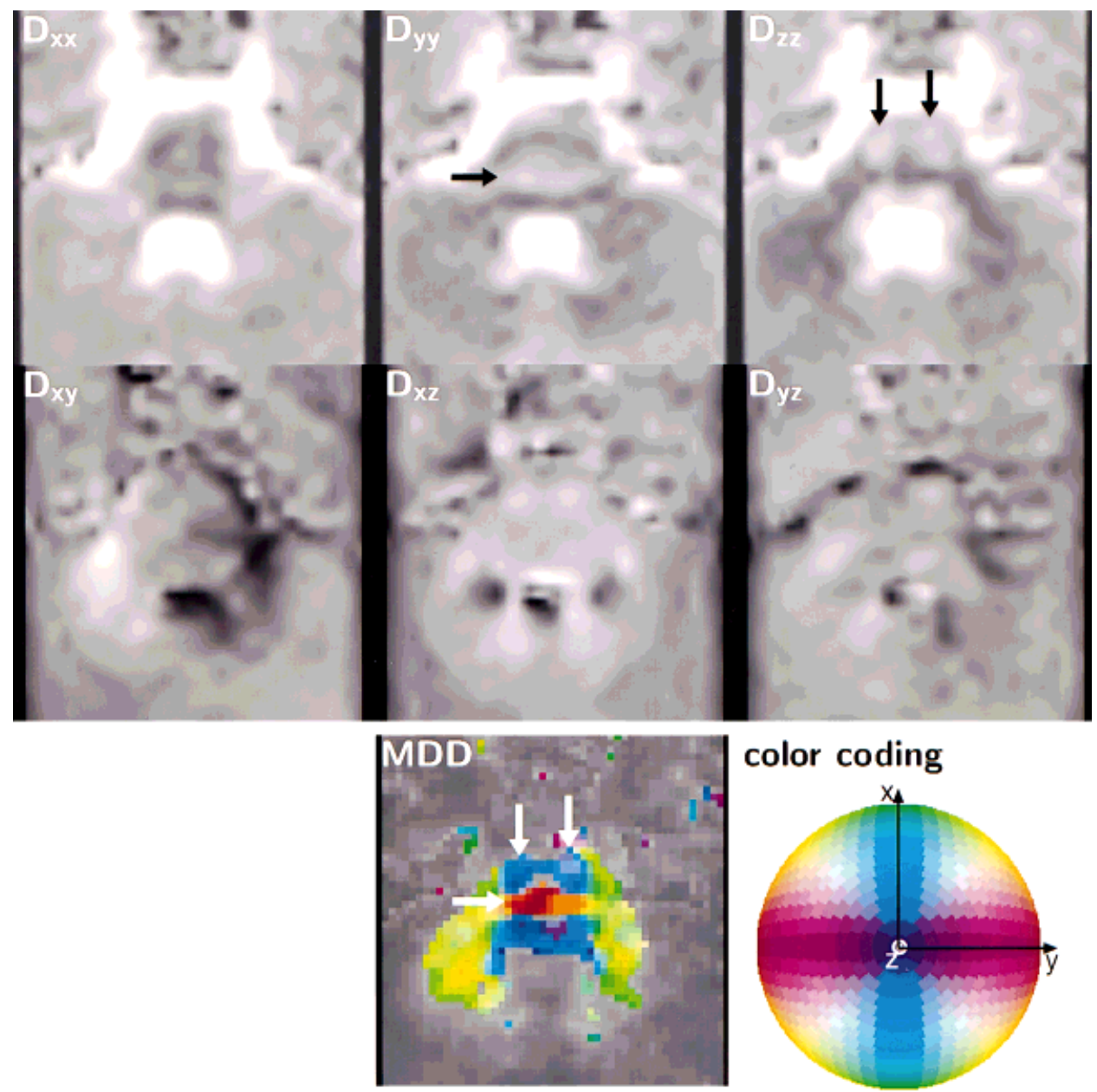

Figure 5. Diffusion tensor mapping of the human brain stem (same data and parameters as in Fig. 4). The maps represent the six elements of the diffusion tensor, a color-coded map of the main diffusion direction (MDD), and the color scale used for directional encoding.

in the color map). Pertinent contributions to the $D_{z z}$ map represent fibers that pass the pons on their way from the cerebrum to the spine (or vice versa), forming the pyramidal tracts. In contrast, the primary diffusion direction in the central region marked by a horizontal arrow is left-right (red to orange), as derived from the $D_{y y}$ map. It indicates the prevalence of fibers connecting to the left and right penduncles of the cerebellum, which -in neighboring regions-exhibit diffusion components in the top-bottom direction (green to yellow). Figure 6 shows a series of consecutive RA and MDD maps from sequentially acquired sections in the human brain stem that may be exploited for fiber tracking in clinical conditions such as traumatic lesions, neoplasms, and neurodegenerative disorders.

\section{DISCUSSION}

Apart from a tremendous motion robustness, the most attractive feature of DW single-shot LSI is its insensitivity to geometric distortions and signal losses in the presence of susceptibility differences, which, of course, is based on the acquisition of RF refocused stimulated echoes. Pertinent characteristics are important when attempting to map diffusion tensor properties in body regions that are difficult or even impossible to study with EPI. In this respect, the present work demonstrates access to the pyramidal tracts in the human brain stem. The example supports the notion that diffusion tensor mapping provides a unique contrast mechanism reflecting the microstructure of the tissue under investigation. Such information relates to the orientation of nerve fibers or, more generally, of bundles of myelinated axons. Although initial studies of white matter in newborns suggest that diffusion tensor mapping may become a sensitive tool for detecting disturbances of brain myelination during development, eg, by its potential to detect inappropriate diffusion anisotropy (5), careful clinical investigations are warranted to prove a diagnostic benefit.

A limitation of DW single-shot LSI is its lower SNR compared with Fourier imaging. However, at least some of the theoretical loss can be compensated for by a much smaller receiver bandwidth, as commonly used for EPI. Nevertheless, the coverage of larger volumes within short measuring times is restricted, as extensions to interleaved studies of multiple sections are at the expense of in-plane resolution (8). The technique is therefore expected to find niche applications in which 


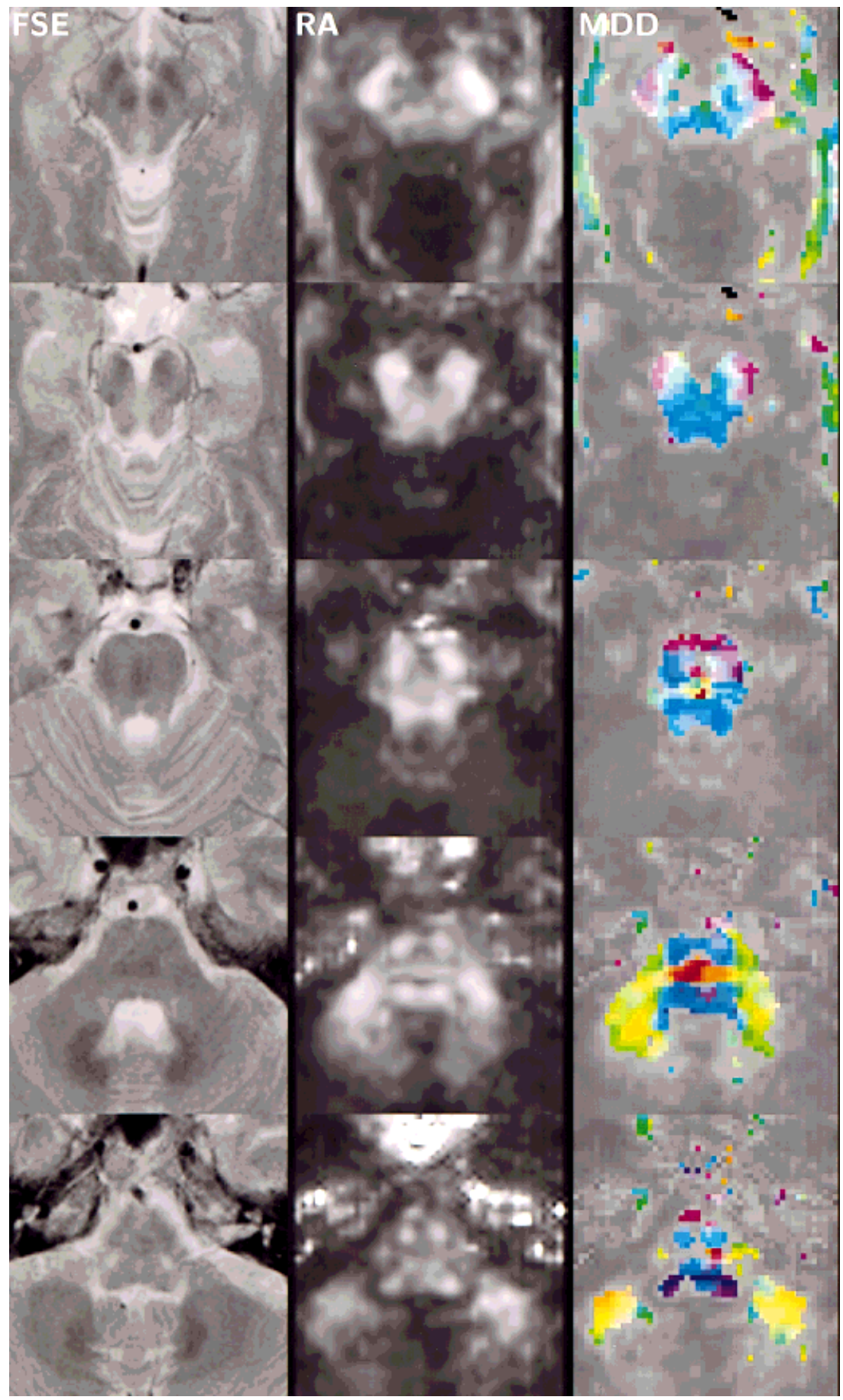

Figure 6. Left: Fast spin-echo images. Middle: Corresponding relative anisotropy (RA) maps. Right: Color-coded maps of the main diffusion direction (MDD) in five sections covering the brain stem. The total measurement time was 9 minutes (For other parameters see Fig. 4.)

its motion robustness and $T 2 *$ insensitivity prove superior to EPI.

A promising feature of DW single-shot LSI is that it profits from (very) high magnetic fields: because $T 1$ relaxation times increase with field strength, the time available for STE acquisitions of individual lines is prolonged. This can be used to improve either the receiver bandwidth, ie, the SNR, or the FOV or spatial resolution by adding more lines. The advantageous field strength dependence is opposite to that of EPI, as the $T 2 *$ reduc- tion at higher fields results in extended gradient requirements, increased bandwidths, and even more pronounced susceptibility artifacts.

\section{REFERENCES}

1. Pierpaoli C, Basser PJ. Toward a quantitative assessment of diffusion anisotropy. Magn Reson Med 1996;36:893-906.

2. Pierpaoli C, Jezzard P, Basser PJ, Barnett A, Di Chiro G. Diffusion tensor MR imaging of the human brain. Radiology 1996;201:637648. 
3. Conturo TE, Lori NF, Cull TS, et al. Tracking neuronal fiber pathways in the living human brain. Proc Natl Acad Sci USA 1999;96: 10422-10427.

4. Huppi PS, Maier SE, Peled S, et al. Microstructural development of human newborn cerebral white matter assessed in vivo by diffusion tensor magnetic resonance imaging. Pediatr Res 1998;44:584-590.

5. Warach S, Chien D, Li W, Ronthal M, Edelman RR. Fast magnetic resonance diffusion-weighted imaging of acute human stroke. Neurology 1992;42:1717-1723.
6. Gudbjartsson H, Maier SE, Mulkern RV, et al. Line scan diffusion imaging. Magn Reson Med 1996;36:509-519.

7. Schick F. SPLICE: sub-second diffusion-sensitive MR imaging using a modified fast spin-echo acquisition mode. Magn Reson Med 1997; 38:638-644.

8. Finsterbusch J, Frahm J. Diffusion-weighted single-shot line scan imaging of the human brain. Magn Reson Med 1999;42:772-778.

9. Frahm J, Haase A, Matthaei D, Merboldt KD, Hänicke W. Rapid NMR imaging using stimulated echoes. J Magn Reson 1985;65:130-135. 